\title{
Visual detection of line segments: Two exceptions to the object superiority effect
}

\author{
RAYMOND KLEIN \\ Dalhousie University, Halifax, Nova Scotia, B3H 4J1, Canada
}

\begin{abstract}
Boundary conditions for the object superiority effect IOSE, target lines embedded in meaningful drawings are more accurately identified than the same targets embedded in nonmeaningful drawings; Weisstein \& Harris, 1974) were sought by examining two variables known to affect the word superiority effect. A robust OSE was obtained in both two- and four-alternative tasks with no mask or speed requirement. The OSE was eliminated by either a random masking stimulus composed of dots or lines (Experiment 1) or by requiring speeded identification responses (Experiment 2). An information processing model is proposed to account for the OSE, as well as the exceptions reported herein. It is shown that this model can be modified to deal with the analogous word superiority effect.
\end{abstract}

It has been demonstrated that identification of a briefly flashed line segment is more accurate when the line is part of a drawing that appears unitary and three-dimensional than when the line is embedded in a variety of less coherent figures (Weisstein \& Harris, 1974; Williams \& Weisstein, 1976; Womersley, 1977; for a review, see Weisstein \& Maguire, in press). In the original study of this phenomenon (Weisstein \& Harris, 1974), the subject's task was to identify which one of four oblique target lines was present in a near-threshold drawing. On each trial, a target line was embedded in a particular context, the combined stimulus was presented briefly and was followed immediately by a masking stimulus. Identification accuracy was directly related to the meaningfulness (three-dimensionality, familiarity, coherence) of the drawings. By naming this phenomenon the "object superiority effect" (OSE), Weisstein and Harris were calling attention to the analogous "word superiority effect" (WSE)': the identification of letters embedded in words is superior to that of letters alone or to letters embedded in nonwords (Reicher, 1969; Wheeler, 1970; for a review, see Baron, in press). The companion phenomena have an important implication for models of perception. They suggest that the detection of simple features or elements (e.g., lines, letters) is influenced by representational processes

This research was supported by grants from the National Research Council of Canada and the Dalhousie Development Fund, and was presented at the Eastern Psychological Association, April 1976, New York. I would like to thank Ed Hansen for running subjects and John Barresi for his comments on an earlier draft of this paper. Requests for reprints should be addressed to Raymond Klein, Department of Psychology, Dalhousie University, Halifax, Nova Scotia, B3H 4J1, Canada. sensitive to properties of the whole stimulus pattern.

Our studies of object superiority have revealed two situations in which the normally robust effect is absent. The variables which eliminate the OSE should help to specify boundary conditions, for the processing mechanism that is presumed to underlie the phenomenon. In this report, I will describe these exceptional cases, outline an information processing model which accounts for the OSE and the present findings, and explore the applicability of such a model to the WSE.

\section{EXPERIMENT 1}

The first experiment was designed primarily to replicate the OSE. With this goal in mind, the backgrounds that produced the highest and lowest levels of performance in the Weisstein and Harris experiment were employed. If the word and object superiority effects are analogous, then one would expect to find parallel boundary conditions for the two phenomena. Since there is evidence suggesting that the presence of a masking stimulus may be important for obtaining superiority of words over single letters (Johnston \& McClelland, 1973; Juola, Choe \& Leavitt, Note 1), both mask and no-mask conditions were included.

\footnotetext{
Method

Apparatus and Stimuli. A three-field Scientific Prototype tachistoscope was use to present the stimuli. Eight different drawings were generated by placing one of four oblique lines (targets) in corresponding locations in the two backgrounds (see Figure 1), which will be referred to as meaningful and nonmeaningful. A masking stimulus consisted of a random arrangement of straight lines that were equal in length to the lines in the two backgrounds, enclosed in a square border (see
} 

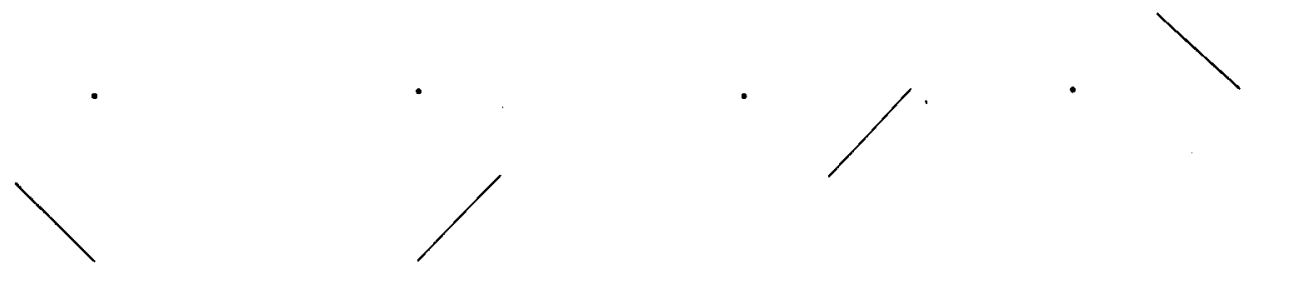
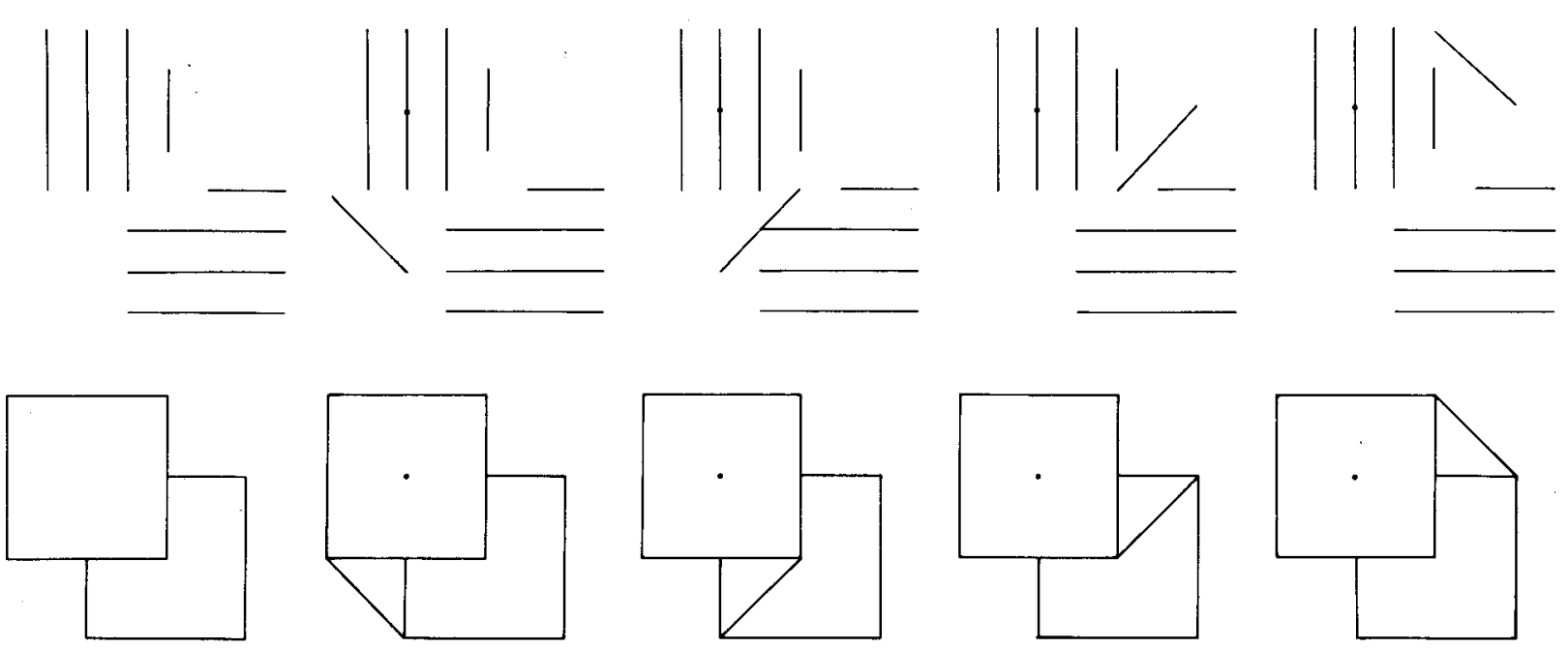

Figure 1. Targets (top row), contexts (left column), and eight stimuli made be embedding the four targets in the two contexts. Middle row shows the nonmeaningful stimuli and bottom row shows meaningful stimuli.

Figure 2a). These stimuli were drawn (black on white) by an artist and photographed and printed on $4 \times 6$ in. glossy paper. When viewed in the $T$-scope, the target lines subtended a visual angle of $.5^{\circ}$. One field of the T-scope was used to display the fixation stimulus (a small black dot in the center of a white field), while the remaining fields were used to display the drawing and the poststimulus (mask or blank). The luminances of the three fields were equal at $10 \mathrm{fL}$.

Procedure. There were two presentation conditions. In the no-mask condition the subject viewed the fixation field for $500 \mathrm{msec}$. This was followed immediately by one of the eight drawings, which was presented for $20-42 \mathrm{msec}$, depending upon the subject's performance level. Immediately after the drawing, a blank field was presented for $750 \mathrm{msec}$. In the mask condition the sequence was the same, except that the drawings were displayed for a longer period $(65-300 \mathrm{msec})$ and were followed by the mask for $750 \mathrm{msec}$. At the end of a sequence, the subject examined a sheet showing each of the four target lines in relation to the fixation point and was required to state which one had been presented.

The subjects were run for 4 days. The first part of each day was devoted to determining exposure durations for the two pre- sentation conditions that would keep performance at about $70 \%$ $80 \%$ correct (uncorrected). The mask and no-mask conditions were run in separate 64-trial blocks, and the eight different stimuli were presented randomly within a block. On each of 4 days, the subjects were tested once in the mask condition and once in the no-mask condition. The order of mask and nomask blocks was varied within and between subjects. Over the 4 days of testing, each subject was tested on 128 trials in each of the 4 basic conditions (meaningfulness of background crossed with mask vs. no mask).

Subjects. Four Dalhousie University students (two graduate and two undergraduate) were paid $\$ 2$ per session. All subjects were naive concerning the nature of the effect being explored.

\section{Results and Discussion}

The performance data shown in Table 1 reveal a striking interaction $[F(1,3)=24.4, p<.025]$ : in the mask condition there is no difference between the meaningful and nonmeaningful drawings, while in the no-mask condition there is a large OSE
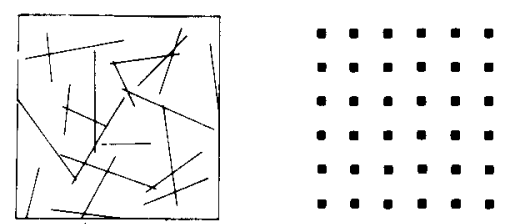

a b

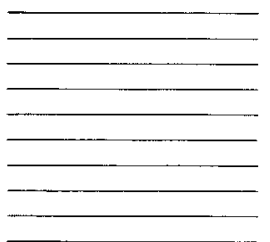

c
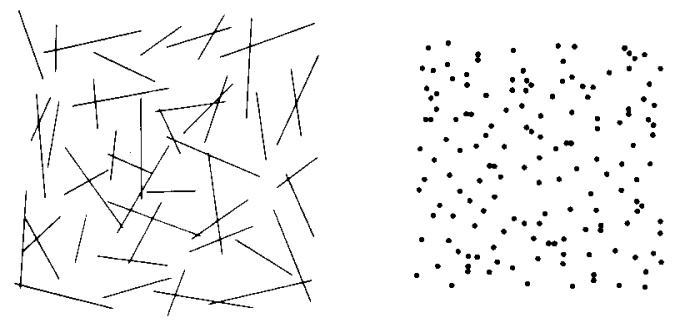

Figure 2. Masking stimuli from Experiment 1 (a), Weisstein and Harris (1974) (b), Womersley (1977) (c), and Experiment 1a (d,e). 
Table 1

Probability Correct ${ }^{2}$ for Each Subject and Condition of Experiment 1

\begin{tabular}{ccccc}
\hline & \multicolumn{2}{c}{ Mask } & & \multicolumn{2}{c}{ No Mask } \\
\cline { 2 - 5 } & $\square$ & $\bar{E}_{1}$ & & $\square$ \\
S1 & 63.4 & 83.3 & 85.3 & 48.8 \\
S2 & 81.2 & 69.9 & 52.1 & 18.0 \\
S3 & 72.8 & 82.2 & 81.3 & 58.4 \\
S4 & 55.2 & 45.9 & 82.4 & 41.4 \\
Mean & 68.1 & 70.3 & 75.2 & 41.7 \\
\hline
\end{tabular}

$[\mathrm{T}(3)=8.43, \mathrm{p}<.01$, two-tailed $]$.

The magnitude and consistency of the OSE obtained in the no-mask condition constitutes an excellent replication. Nevertheless, Weisstein and Harris (1974) and Womersley (1977) obtained the effect using a masking stimulus, while in the present study the masking stimulus eliminated the effect. Resolution of this discrepancy probably lies in differences between the masking stimuli used in the studies. Weisstein and Harris used a mask (Figure 2b) that was redundant, did not overlap the stimulus, and had no structural elements in common with the stimulus. Womersley's mask was also redundant (Figure 2c). In contrast, the mask used in Experiment 1 (Figure 2a) was random, did overlap the stimulus, and was composed of similar structural elements. Recent evidence (Berbaum, Weisstein, \& Harris, 1975) suggests that the OSE may depend in part upon a perceptual system that relies upon angles to construct three-dimensional representations. The presence of many random angles in the randomline mask may have interfered with the operation of this system. An alternative explanation concerns the presence of the square border in the randomline mask. This border may have selectively interfered with the meaningful background which contained two overlapping squares.

In order to determine whether the powerful effect of the random-line mask was due to the presence of angles, a square border, or just to randomness, three of the four subjects from Experiment 1 were tested in six additional sessions (Experiment 1a). The first four of these sessions used two masking stimuli; one was composed of random lines with no border, the other was composed of random dots (Figures $2 \mathrm{~d}$ and $2 \mathrm{e}$ ). To be sure that the OSE had not disappeared as a result of practice, these subjects were tested in two further sessions with no mask and the line mask (Figure 2d). Results from this control experiment are shown in Table 2. There was no significant effect of meaningfulness in the two mask conditions. More important, it can be seen that the two random masks are behaving in exactly the same manner. Results from the last two sessions show that the object superiority effect is still present $[\mathrm{T}(2)=9.51, \mathrm{p}<.02$, two-tailed] when no mask is used. ${ }^{3}$ These additional observations imply that it is randomness of the masking stimulus that eliminates the advantage for meaningful material. It seems reasonable to conclude that any "good" pattern mask, i.e., one which tends to destroy the integrity of the pattern, will disrupt or eliminate the OSE. ${ }^{4}$ This descriptive statement leaves two related problems unresolved. First, why does a random mask eliminate the object superiority effect? Second, recall that the WSE is typically obtained with a random mask and occasionally absent without one. In the face of the contradictory effects of random masking stimuli, is it still plausible to seek common explanatory principles for object and word superiority? We will return to these issues in the general discussion.

\section{EXPERIMENT 2}

Existing studies of the OSE have naturally emphasized accuracy rather than speed of response. Using the powerful speed-accuracy tradeoff paradigm (Wickelgren, 1977), Polf (1976) has shown that the advantage for words over single letters is not obtained at short latencies (in fact, the effect was reversed). Still seeking parallel boundary conditions, a second experiment was conducted to determine whether the OSE would be eliminated by requiring speeded identification responses.

Table 2

Probability Correct ${ }^{2}$ for Each Subject and Condition of Experiment 1a

\begin{tabular}{|c|c|c|c|c|c|c|c|c|}
\hline & \multicolumn{4}{|c|}{ Sessions 1-4 } & \multicolumn{4}{|c|}{ Sessions 5-6 } \\
\hline & \multicolumn{2}{|c|}{ Line Mask } & \multicolumn{2}{|c|}{ Dot Mask } & \multicolumn{2}{|c|}{ Line Mask } & \multicolumn{2}{|c|}{ No Mask } \\
\hline & $\square$ & $\equiv_{\|}$ & $\square$ & $\equiv_{\text {}} \|$ & & $\equiv_{1 \mid}$ & & $\equiv_{, \|}$ \\
\hline $\begin{array}{l}\text { S1 } \\
\text { S2 } \\
\text { S3 }\end{array}$ & $\begin{array}{l}51.5 \\
67.7 \\
48.0\end{array}$ & $\begin{array}{l}63.9 \\
33.3 \\
50.0\end{array}$ & $\begin{array}{l}65.2 \\
72.9 \\
48.0\end{array}$ & $\begin{array}{l}73.2 \\
50.0 \\
46.9\end{array}$ & $\begin{array}{l}62.5 \\
81.2 \\
40.3\end{array}$ & $\begin{array}{l}58.4 \\
35.5 \\
45.9\end{array}$ & $\begin{array}{l}79.2 \\
75.1 \\
83.6\end{array}$ & $\begin{array}{l}35.5 \\
34.0 \\
52.1\end{array}$ \\
\hline Mean & 55.7 & 49.1 & 62.0 & 56.4 & 61.3 & 46.5 & 79.3 & 40.5 \\
\hline
\end{tabular}

Note-See Figures $2 d$ and $2 c$ for line and dot masks. 


\section{Method}

The apparatus and stimuli were the same as in the first experiment, with the following exceptions: (1) the onset of the stimulus started a digital timer, (2) the subject's keypress response terminated the timer, (3) no mask was used, and (4) the fixation stimulus was presented for $1.5 \mathrm{sec}$. The procedure was also similar, except that, to simplify the subject's task, the stimulus duration was kept constant at $125 \mathrm{msec}$ and the subject made a two-choice response concerning the slope of the target line (instead of a four-choice judgment of slope and location). Subjects were instructed to respond as quickly as possible, without sacrificing accuracy. Six naive subjects were tested in two 1-h sessions, providing 128 trials in each condition.

\section{Results and Discussion}

Accuracy and latency data for each condition are shown in Table 3. There is clearly no difference between the two backgrounds when accuracy is considered. The OSE has once again been eliminated. Furthermore, latencies are actually faster for the nonmeaningful background $[\mathrm{T}(5)=7.82, \mathrm{p}<.001$, two-tailed].

It is tempting to conclude that the variable underlying the elimination of the OSE in this experiment is the speeded response required of the subject. Two other procedural changes, however, could have been responsible. In Experiment 2, the subjects made two-alternative slope judgments, while in Experiment 1 there were four alternatives, with subjects implicitly judging both slope and location. Secondly, the stimulus duration was relatively long $(125 \mathrm{msec})$ in Experiment 2 and much closer to the duration used in the masking condition of Experiment 1 , in which the effect was not obtained. Although a mechanism is not obvious, it is possible that this common factor is responsible for eliminating the effect in both experiments. To determine if these procedures play some role in eliminating the OSE, two control experiments were run. In the first (2a), two subjects made unspeeded slope judgments under the no-mask conditions of Experiment 1 . Under these conditions, a typical OSE was obtained ( $71 \%$ for meaningful and $40 \%$ for nonmeaningful stimuli). In the second control experiment (2b), two subjects were tested in the speeded response para-

Table 3

Reaction Time and Probability Correct $^{2}$ for Each Subject and Condition of Experiment 2

\begin{tabular}{|c|c|c|c|c|}
\hline & \multicolumn{2}{|c|}{$\square$} & \multicolumn{2}{|c|}{$\overline{\overline{\bar{F}}}_{\|} \|$} \\
\hline & RT & $\begin{array}{l}\text { Percent } \\
\text { Correct }\end{array}$ & RT & $\begin{array}{l}\text { Percent } \\
\text { Correct }\end{array}$ \\
\hline $\begin{array}{l}\text { S1 } \\
\text { S2 } \\
\text { S3 } \\
\text { S4 } \\
\text { S5 } \\
\text { S6 }\end{array}$ & $\begin{array}{l}528 \\
535 \\
490 \\
558 \\
507 \\
517\end{array}$ & $\begin{array}{l}87.6 \\
95.4 \\
79.8 \\
48.6 \\
76.6 \\
81.4\end{array}$ & $\begin{array}{l}502 \\
506 \\
473 \\
531 \\
466 \\
497\end{array}$ & $\begin{array}{l}90.8 \\
95.4 \\
72.0 \\
56.4 \\
89.2 \\
89.2\end{array}$ \\
\hline Mean & 523 & 78.2 & 496 & 82.2 \\
\hline
\end{tabular}

digm of Experiment 2 with a much shorter $(50 \mathrm{msec})$ exposure duration. As in Experiment 2, there was no OSE (70\% and $72 \%$ correct for meaningful and nonmeaningful, respectively) and RTs were faster for the nonmeaningful stimuli (504 and $484 \mathrm{msec}$ ).

These control experiments demonstrate that neither the long duration of the stimulus nor the slope judgment were responsible for eliminating the OSE in Experiment 2. By the process of elimination, we are forced to conclude that it is the latency of the response which is crucial: at short latencies, the object is not superior. Furthermore, the significantly faster responses to the nonmeaningful stimuli suggest that speed stress actually reverses the OSE.

\section{GENERAL DISCUSSION}

These experiments reveal two situations in which a normally robust object superiority effect is eliminated. Any satisfactory account of object superiority must describe not only the perceptual mechanisms which produce the effect, but also how a random pattern mask and speed stress interact with these mechanisms to eliminate it. One such model is presented below. ${ }^{5}$

\section{Information Processing Model of Object Superiority}

The basic assumptions of this model are that more than one processing level or system may contribute to identification of a visual target (e.g., line segment), and that a central decision mechanism may consult these systems for task-relevant information accruing in real time. For the sake of simplicity, we may assume that there are two systems. (See Hock, 1973, for evidence supporting a similar distinction between perceptual systems.) The feature system is sensitive to elementary features (orientation, location, etc.) of the visual stimulus. Except for local effects such as simultaneous masking, the response of this system to the target element is the same regardless of the surrounding context. The object system does not represent individual features, but instead integrates the elements of the stimulus to represent global properties in a three-dimensional medium. This system is selectively activated by object-like stimuli that are threedimensional, coherent, and/or meaningful. ${ }^{6}$

As described so far, this general model can account for the OSE, since both representational systems will contribute information about the target element embedded in a meaningful context, but only the feature system will contribute with nonmeaningful contexts. To accomodate the effect of speed stress, it is further assumed that the object system does not provide usable target identity information at short latencies (see Figure 3). This might be due to the length of time required to activate the object 


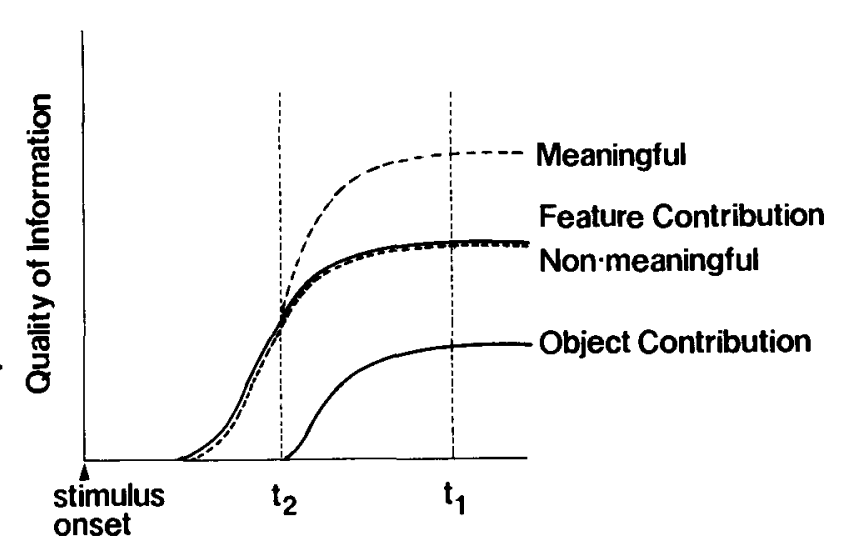

Time

Figure 3. Quality of information regarding target identity is shown for both the object and feature systems as a function of time. With "slow" responses (in the vicinity of $t_{1}$ ), an OSE will be obtained because both systems will contribute information when the meaningful stimulus is presented, while for the nonmeaningful stimulus only the feature system will make a contribution. Any variables (e.g., speed stress) that cause the decision mechanism to consult these representational systems earlier than $t_{2}$ will eliminate the effect.

system or to the time required to decompose or scan an object representation for the presence of a particular target element. The effect of the random mask can be accommodated in either of two ways. First, it might be assumed that the mask destroys any representation in the object system. Second, the mask might force the subject to generate a response before the object system has had time to contribute any identity information (i.e., it may operate by inducing speed stress).

If we limit our scope to the OSE, the latter explanation is preferred, since given the known effects of speed stress (Experiment 2), it seems more parsimonious. On the other hand, faith in the object-word superiority parallelism seems to logically exclude the speed-stress explanation for the masking effect, since the WSE, also eliminated by speed stress, is obtained with a random mask. Perhaps we should hold judgment for the time being and examine the applicability of our model to the WSE.

\section{Extension of Model to the Word Superiority Effect}

A similar explanation for the superiority of words over single letters and nonwords is supported by several studies (Hawkins, Reicher, Rogers, \& Peterson, 1976; Johnston \& McClelland, 1973; Mezrich, 1973; Polf, 1976). Words, nonwords, and letters are assumed to activate a system representing the physical properties of the stimulus with roughly equal facility; words, however, are more likely to activate deeper (Craik \& Lockhart, 1972) or more abstract (Posner, 1969) systems representing name and semantic information. ${ }^{7}$ These abstract (derived) representations will aid in the forced-choice judgment of letter identity as long as the subject is given sufficient time (Polf, 1976) and incentive (Hawkins, et al., 1976) to use them. The parallel seems to break down when we consider the effects of the random masks: Why is object superiority eliminated by a mask, while word superiority is not? The key to this discrepancy lies in the relationship between the masking stimulus and the representational systems presumed to underlie the effects. Once name and semantic representations have been activated, they will be relatively immune from the destructive force of a random pattern mask. On the other hand, a visual pattern mask will interfere with physical representations: thus, with verbal materials, its overall effect is to enhance the relative contribution of the representational systems that mediate the WSE. ${ }^{8}$ With object superiority, however, the "deeper" representational system is assumed to be a visual system. Thus, while the random mask interferes equally with the feature representations of the visual stimuli, its deleterious effects may prevent the object system from making any significant contribution. The fact that the object superiority effect is obtained with regular masks, which do not destroy the integrity of the pattern, provides some support for this view.

\section{SUMMARY AND CONCLUSION}

In this study, we have begun a research strategy of exploring the boundary conditions on the object superiority effect and we have compared and contrasted these with similar conditions on the word superiority effect. We have uncovered two situations in which the OSE is eliminated. A general information processing model was proposed to accont for the OSE as well as these exceptions, and this general model was shown to be applicable to the WSE. We feel that further comparisons of these analogous phenomena will provide fruitful information on the role of meaningfulness in perception. A particularly important issue is the degree to which subjects can exert strategic control over the utilization of the various representational systems that may be activated by a stimulus. Hawkins et al. (1976) and Mezrich (1973), for example, have demonstrated flexible use of name and semantic representation in the WSE. While the proposed model is consistent with the evidence, it is by no means necessitated by it. We believe that application of powerful chronometric methods such as the speed-accuracy tradeoff paradigm (Wicklegren, 1977) should provide useful converging evidence on the validity of the dynamic information processing 
assumptions made by the model. It will also be necessary to attempt to relate this type of analysis to the interesting chronometric method that has been pursued by Williams and Weisstein (1976). Lastly, whether the proposed model is correct or not, any model of the OSE, to be satisfactory, must explain the exceptions reported here.

\section{REFERENCE NOTE}

1. Juola, J., Choe, C., \& Leavitt, D. A reanalysis of the word-superiority effect. Paper presented at the 15th Annual Meeting of the Psychonomic Society, Boston, Massachusetts, 1974.

\section{REFERENCES}

BARON, J. The word superiority effect. In W. K. Estes (Ed.), Handbook of learning and cognitive processes, 5. Hillsdale, N.J: Erlbaum, in press.

Berbaum, K., Weisstein, N., \& Harris, C. A. Certain types of vertices and line detection. Bulletin of the Psychonomic Society, 1975, 6, 418. (Abstract)

CRaIK, F. M., \& Lockhart, R. S. Levels of processing: A framework for memory research. Journal of Verbal Learning \& Verbal Behavior, 1972, 11, 671-784.

Hawkins, H., Reicher, G., Rogers, M., \& Peterson, L. Flexible coding in word recognition. Joumal of Experimental Psychology: Human Perception and Performance, 1976, 2, 380-385.

Hock, H. The effects of stimulus structure and familiarity on same-different comparison. Perception \& Psychophysics, 1973. $14,413-420$.

Johnston, J. C., \& MCClelland, J. L. Visual factors in word perception. Perception \& Psychophysics, 1973, 14, 365-370.

Mezrich, J. The word superiority effect in brief displays: Elimination by vocalization. Perception \& Psychophysics, 1973, $13,45-48$.

PoLF, J. The word superiority effect: A speed accunacy analysis and test of a decoding hypothesis. Unpublished doctoral dissertation, University of Oregon, 1976.

PosNer, M. I. Abstraction and the process of recognition. In G. Bower (Ed.), The psychology of learning and motivation (Vol. 3). New York: Academic Press, 1969.

ReICHER, G. Perceptual recognition as a function of meaningfulness of stimulus material. Journal of Experimental Psychology, 1969, 81, 274-280.

Rumelanat, D. E., \& Siple, P. The process of recognizing tachistoscopically presented words. Psychological Review, 1974, 81, 99-118.
WEISSTEIN, N., \& HARRIS, C. S. Visual detection of line segments: An object-superiority effect. Science. 1974, 186, 752-755.

Weisstein, N., \& Maguire, W. Computing the next step: Psychophysical measures of representation and interpretation. In E. M. Riseman and A. R. Hanson (Eds.), Computer vision systems. New York: Academic Press, in press.

WhEEler, D. Processes in word recognition. Cognitive Psychology, 1970, 1, 59-85.

Wickelgren, W. A. Speed-accuracy tradeoff and information processing dynamics. Acta Psychologica, 1977, 41, 67-85.

Williams, A., \& Weisstein, N. The time course of object superiority. Bulletin of the Psychonomic Society, 1976, 8, 260. (Abstract)

Womersley, M. A contextual effect in feature detection with application of signal detection methodology. Perception \& Psychophysics, 1977, 21, 88-92.

\section{NOTES}

1. We will use the abbreviations OSE and WSE to refer to the object and word superiority effects, respectively. WSE will refer to the advantage of words over nonwords and single letters, these situations being distinguished only where necessary.

2. All proportions reported as data have been adjusted by the following formula: $\mathrm{P}_{a}=\left(\mathrm{P}_{\circ}-\mathrm{P}_{\mathrm{g}}\right)\left(1-\mathrm{P}_{a}\right) \mathrm{P}_{a}=$ obtained proportion correct, $P_{s}=$ proportion correct based on chance responding (e.g., $\mathrm{P}_{2}=.25 \mathrm{w} / 4$ equally likely alternatives). By this procedure, $P_{*}=0$ represents chance performance regardless of the number of alternatives.

3. The nonsignificant effect of meaningfulness with masks (in all six sessions) is due to one subject, for whom the masks do not seem to eliminate the OSE.

4. Weisstein (personal communication) has come to a similar conclusion.

5. See Johnston and McClelland (1973) and Hawkins et al. (1976) for discussions of related models for the WSE.

6. Which of these properties is sufficient to produce an OSE is not clear, though three-dimensionality is supported by Womersley's (1977) Experiment 2.

7. This is not to rule out the role visual processes such as feature redundancy (e.g., Rumelhart \& Siple, 1974) may play in some circumstances.

8. In the absence of a mask, in some conditions the relative contributions of name and semantic representations may be so small as to provide no incentive for their use.

(Received for publication April 3, 1978; accepted June 13, 1978.) 